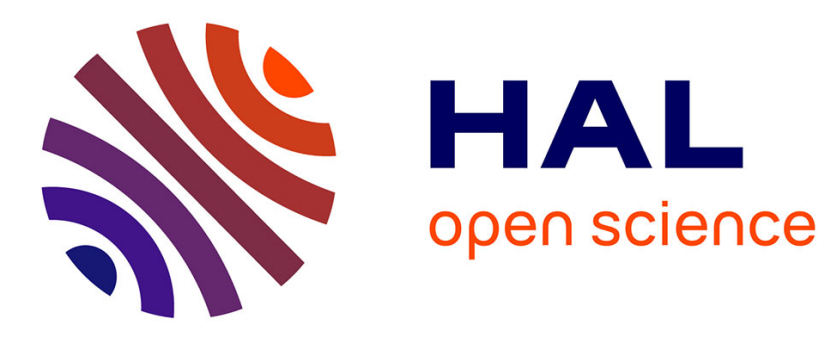

\title{
Le destin singulier de Marx dans la science économique en France
}

Thierry Pouch

\section{To cite this version:}

Thierry Pouch. Le destin singulier de Marx dans la science économique en France. Jean-Numa Ducange; Antony Burlaud. Marx, une passion française, éditions La Découverte, 2018, coll. "Recherches. hal-02084068

\section{HAL Id: hal-02084068 \\ https://hal.univ-reims.fr/hal-02084068}

Submitted on 9 Apr 2019

HAL is a multi-disciplinary open access archive for the deposit and dissemination of scientific research documents, whether they are published or not. The documents may come from teaching and research institutions in France or abroad, or from public or private research centers.
L'archive ouverte pluridisciplinaire HAL, est destinée au dépôt et à la diffusion de documents scientifiques de niveau recherche, publiés ou non, émanant des établissements d'enseignement et de recherche français ou étrangers, des laboratoires publics ou privés. 
SOUS LA DIRECTION DE

Jean-Numa Ducange et Antony Burlaud

Depuis le début des années 1980, on a assisté à un redéploiement considérable de la recherche en sciences humaines et sociales : la remise en cause des grands systèmes théoriques qui dominaient jusqu'alors a conduit à un éclatement des recherches en de multiples champs disciplinaires indépendants, mais elle a auss permis d'ouvrir de nouveaux chantiers théoriques. Aujourd'hui, ces travaux commencent à porter leurs fruits : des paradigmes novateurs s'élaborent, des liens inédits sont établis entre les disciplines, des débats passionnants font jour. Mais ce renouvellement en profondeur reste encore dans une large mesure peu visible, car il emprunte des voies dont la production éditoriale traditionnelle rend difficilement compte. L'ambition de la collection « Recherches » est précisémen d'iciement compte. L'ambition de la collection « Recherches » est prément d'accueillir les résultats de cette « recherche de pointe » en sciences humaines et sociales : grâce à une sélection éditoriale rigoureuse (qui s'appuie notamment sur l'expérience acquise par les directeurs de collection de La Découverte), elle
publie des ouvrages de toutes disciplines, en privilégiant les travaux transmultidisciplinages de toutes disciplines, en privilégiant les travaux trans- et multidisciplinaires. Il s'agit principalement de livres collectifs résultant de programmes à long terme, car cette approche est incontestablement la mieux à même de rendre compte de la recherche vivante. Mais on y trouve aussi des ouvrages d'auteurs (thèses remaniées, essais théoriques, traductions), pour se faire l'écho de certains travaux singuliers.

Avec le soutien du GRHis-Rouen, de l'IRIHS-Rouen, du CGC-Dijon et de la Fondation Gabriel Péri.

Éditions La Découverte

9 bis, rue Abel-Hovelacque

Paris XIII ${ }^{e}$

2018 
Le destin singulier de Marx dans la science économique en France

Thierry Pouch

Une grande majorité d'économistes français - ceux du mainstream - ne célébrera pas le bicentenaire de la naissance de Karl Marx en 2018. S'y aventurer, ce serait renoncer à stigmatiser un penseur dont l'œuvre est à leurs yeux a-scientifique, et qui, par surcroît, a été vaincu par l'histoire, comme semble le démontrer l'effondrement du bloc soviétique. Pour les économistes hétérodoxes en revanche, le « surgissement fantomal » (J. Derrida) de Marx, à l'occasion de cet anniversaire, offre l'occasion de rappeler que Karl Marx fut et demeure une source fondamentale d'inspiration pour comprendre les ressorts du capitalisme, et en critiquer tant le mode de fonctionnement que les conséquences sociales. Pour ces économistes hétérodoxes, Marx a décortiqué la logique et la dynamique du capitalisme, en a mis au jour les contradictions tout en traçant des perspectives révolutionnaires. La grande crise capitaliste qui s'est enclenchée en 2007 avec l'éclatement de la bulle immobilière aux États-Unis n'a pas permis de restaurer la place de Marx dans les cursus de sciences économiques des universités françaises, et moins encore dans les revues scientifiques traitant d'économie. Dans le champ de l'économie, Marx reste marginal. Mais, comme on va le voir, il n'en a pas toujours été ainsi. Le marxisme était parvenu à s'implanter dans les universités à partir des années 1950, et à y gagner en audience, avant d'en ressortir une trentaine d'années après.
Les facultés de droit dans les années 1950 :

un lieu hostile devenu perméable à Marx

Rien ne prédisposait ce champ scientifique qu'est l'économie à s'ouvrir à Marx et au marxisme à la toute fin des années 1940. Les frontières de l'université ne se laissent pas pénétrer facilement par un penseur aussi sulfureux et subversif que Marx. Et les facultés de droit, dans lesquelles étaient délivrés des enseignements d'économie, avaient la réputation d'être particulièrement conservatrices. C'est donc en dehors du champ universitaire que se situaient à l'époque les connaisseurs et les diffuseurs de la pensée de Marx, tel Jean Duret.

Un tournant va s'opérer au détour des années 1950 : plusieurs professeurs de la faculté de droit de Paris vont introduire le marxisme dans leurs cours et leurs publications. Jean Lhomme, Jean Marchal, André Piettre figurent parmi les universitaires les plus réceptifs au message marxiste. Selon eux, il est impossible d'enseigner l'économie sans un détour par l'œuvre économique de Marx. Les étudiants ne peuvent en effet aborder les problèmes économiques et sociaux de leur temps en ignorant tout de cet auteur [Marchal, 1955]. Des motivations autant internes qu'externes à l'université expliquent une telle posture intellectuelle. Il s'agit pour ces économistes de faire contrepoids à la montée en puissance de la théorie néoclassique, qui accompagne le déclin de l'école institutionnaliste américaine. Pour eux, les enseignements du désastre occasionné par la crise de 1930 doivent être tirés, et la prétention de l'école néoclassique à dominer le champ de la science économique par la production d'une économie pure, sans institutions ni histoire, doit être combattue. Le recours à Marx constitue une arme dans ce combat. La création en 1950 de la Revue économique - qui devait s'appeler en réalité Revue économique et sociale par ces professeurs d'économie, constitue un signe fort de l'ambition de faire de l'économie une discipline ouverte sur les autres champs, comme la sociologie ou l'histoire [Steiner, 2000]. Dans l'espace des productions scientifiques, cette école de pensée, baptisée "réaliste-sociologique », devient dominante. Elle s'impose dans les universités, et spécifiquement à la faculté de droit de Paris.

Les racines d'un tel positionnement dans le champ de la science économique sont à rechercher du côté du catholicisme social, dont ces professeurs se veulent pour la plupart les dépositaires. C'est pourquoi ils ont tendance à mettre en exergue un Marx humaniste plutôt que révolutionnaire. Mais 
il y a une raison plus directement biographique à l'adhésion au marxisme de ces économistes. Il faut en effet y voir une manière d'effacer leur implication, directe ou indirecte, dans l'idéologie corporatiste des années de la collaboration. Tous les économistes mentionnés ont en effet publié durant les années 1940 des textes traitant des vertus du corporatisme et soulignant l'importance de l'artisanat et de la paysannerie dans le renouveau de la France. Ce fut aussi le cas d'un professeur a priori peu suspect de compromission, tant son œuvre va constituer, plusieurs années après, l'un des principaux vecteurs de l'implantation du marxisme en économie dans les universités françaises. Il s'agit d'Henri Denis. La publication en 1941 de son «Que sais-je ? », intitulé La Corporation, livre de ce point de vue une bonne illustration des engagements et des revirements idéologiques de cette cohorte de professeurs d'économie. Dans un autre ouvrage, Introduction aux problèmes économiques, publié en 1942 dans la collection « Bibliothèque du peuple » des Presses universitaires de France (PUF), Denis avance l'idée que « l'avènement du socialisme consacrerait la ruine de toute civilisation humaine ».

Seule exception à ce tableau, la position d'Henri Bartoli. Ce fils de résistant, intellectuel catholique, proche du courant personnaliste d'Emmanuel Mounier, professeur d'économie à la faculté de Grenoble, puis à Paris a, au contraire de ses collègues, tenté de concilier humanisme et communisme, sans s'être en quoi que ce soit converti à la pensée de Marx. Dans plusieurs ouvrages qui ont contribué à la diffusion de la pensée de Marx en économie, Bartoli indique qu'elle a « restauré la dignité de l'homme », en refusant de faire de celui-ci une marchandise [Bartoli, 1950 ; Pouch, 2001].

Cette entrée de l'économie marxiste à l'université est accueillie avec une dose d'ironie de la part du Parti communiste français (PCF). Mais la création en 1954 de la revue marxiste Économie et Politique à l'initiative du Parti permet la constitution d'une plate-forme où les débats vont pouvoir s'engager entre les économistes humanistes et ceux qui se réclament de Marx comme porteur de l'idée communiste. Henri Denis, fraîchement intéressé par Marx, joue un rôle décisif à Économie et Politique : figure du marxisme français, professeur agrégé à la faculté de droit de Rennes, il est le rédacteur en chef de la revue. Dans plusieurs de ses articles, tout comme dans son ouvrage La Valeur, publié aux Éditions sociales en 1950, Denis fustige l'interprétation de l'œuvre économique de Marx par ses collègues de Paris, mais également par le jésuite Pierre-Yves Calvez. À la «science catholique » doit être opposée une « science marxiste » véritable, et le projet révolutionnaire se substituer au projet de libération de l'homme par Dieu [Denis, $1950 ; 1957]$. Tout un espace de confrontation idéologique se forme à cette époque, permettant aux marxistes de contredire la vision des économistes se réclamant du catholicisme.
Faire du marxisme une science, ou l'apogée du marxisme dans les universités

Dans les années 1960, trois pôles vont se former pour diffuser encore plus largement la question marxiste en économie dans le champ universitaire. Le premier de ces pôles se constitue à partir du courant dit du Capitalisme monopoliste d'État (CME), construction intellectuelle émanant des économistes membres du PCF, qui apparaît lors de la Conférence internationale de Choisy-le-Roi (26 au 29 mai 1966), et va constituer un point d'ancrage fondamental pour beaucoup d'économistes hétérodoxes se réclamant peu ou prou de Marx et se situant dans une perspective de passage au socialisme. Gravitant autour de Paul Boccara, enseignant-chercheur à l'université d'Amiens, ce courant de pensée indique que le capitalisme a pris une forme nouvelle en atteignant le troisième stade de son développement (après les stades primitif-manufacturier et classique-concurrentiel): le stade impérialiste-monopoliste, prenant la forme du CME, lequel doit nécessairement déboucher sur le socialisme, la concentration et la suraccumulation du capital appelant à des nationalisations des monopoles [Collectif, 1971] .

Un second pôle, plus universitaire que le précédent, émerge à peu près simultanément. Il s'agit de l'économie du développement. Ce pôle se forme dans un contexte de décolonisation, et les économistes français qui en sont les initiateurs avancent que la sortie du sous-développement passe par le socialisme. L'ambition de ces économistes est de faire contrepoids au modèle de Rostow, dont l'ouvrage célèbre, Les Étapes de la croissance économique, traduit en français en 1963, avait pour sous-titre : « Manifeste non communiste ». Mais ces débats se radicalisent très vite avec la création, dès 1960, de la revue Tiers-Monde. Deux figures en constituent les coryphées : Gérard Destanne de Bernis, professeur à l'université de Grenoble, et Charles Bettelheim, directeur d'études à la $\mathrm{VI}^{\circ}$ section de l'École pratique des hautes études (EPHE). Le premier produit des analyses - assorties de recommandations au gouvernement algérien - sur le socialisme, et publie, notamment dans Tiers-Monde, mais aussi dans la revue de François Perroux, Économie appliquée, de nombreux articles consacrés à la théorie des « industries industrialisantes » et de la « substitution d'importations». Le second, entré dès l'âge de vingt ans aux Jeunesses communistes, directeur de la collection «Économie et socialisme» chez Maspero, entend montrer que le sous-développement n'est pas un retard à combler selon une logique capitaliste, mais doit être combattu par la voie de la planification socialiste, et s'efforce de déployer une véritable économie politique du socialisme [Bettelheim, 1964; De Bernis, 1966 ; Denord et Zunigo, 2005]. L'empreinte laissée par Charles Bettelheim sur la jeune génération d'économistes est réelle. Gérard Duménil, qui a suivi les séminaires de Bettelheim à l'EPHE, 
en est une illustration. Quelques années plus tard, il forme avec Dominique Lévy un binôme d'économistes dont les analyses du capitalisme et la compréhension de la genèse et des enjeux du néolibéralisme ont, jusqu'à aujourd'hui, grandement contribué à maintenir vivante la pensée de Marx [Duménil et Lévy, 2014].

Reste que l'implantation de Marx et du marxisme dans les universités doit enfin et surtout à l'enseignement d'Henri Denis, qui dispense le cours d'Histoire de la pensée économique à la faculté de droit de Paris. Il convient de rappeler l'impact décisif que ce professeur d'économie au parcours sinueux, muté de Rennes à Paris, a pu avoir sur toute une génération d'étudiants. La publication de son manuel d'Histoire de la pensée économique en 1966, dans publication de son manuel d' Histoire de la pensée économiqué (la collection «Thémis » des PUF) est un moment crucial pour la pénétration du marxisme dans l'université Plus d'un tiers de l'ouvrage est consacré à Marx et au marxisme, Denis égratignant au passage tous les autres courants de la pensée économique (les néoclassiques bien entendu, mais aussi, fait plus surprenant aujourd' hui, les keynésiens et les institutionnalistes); une position très critique qui sera édulcorée dans les éditions suivantes du manuel.

La seconde moitié des années 1960 consacre ainsi la vitalité de Marx et du marxisme dans un champ universitaire, l'économie, qui, auparavant, les avait maintenus à distance. Une génération d'économistes talentueux, fins connaisseurs de la théorie économique, détenant tous les attributs du capital universitaire (thèses d'État, articles dans des revues que l'on nomme aujourd'hui « à comité de lecture », ouvrages et, pour certains d'entre eux, agrégation de l'enseignement supérieur en économie) vont ensuite dans la décennie 1970, propulser Marx et le marxisme sur le devant de la scène universitaire. Deux courants vont apporter une contribution non moins décisive. Le premier regroupe des économistes qui publient dans les Cahiers d'économie politique, revue fondée en 1974, et dont les travaux seront publiés dans la collection «Interventions en économie politique » des Presses universitaires de Grenoble. Carlo Benetti, Suzanne de Brunhoff et Jean Cartelier en sont les figures de proue. Toute leur entreprise est tournée vers l'étude de la structure logique de l'économie politique et la production d'une critique. Il s'agit de convoquer Marx, non plus pour en faire un support d'une quelconque conquête politique, mais pour confronter son œuvre économique (et essentiellement le livre I du Capital, seul livre théorique abouti selon eux), en tant que discours scientifique, à l'économie politique classique et néoclassique, dont ils contestent les catégories supposées naturelles [Benetti, 1976].

Le second groupe se constitue autour d'une autre revue, Critiques de l'économie politique, éditée par Maspero. Pierre Salama, agrégé et professeur des universités, en fut l'un des chefs de file. Ses articles et ouvrages entendent eux aussi contribuer à une critique féroce mais scientifique de l'économie politique. Ce qui le différencie en revanche de ses « camarades» des Cahiers d'économie politique a trait à la fois à leur interprétation de Marx et à leur positionnement dans le champ du militantisme politique. Les économistes groupés autour de Critique de l'économie politique n'entendent pas se limiter au seul livre I du Capital, c'est-à-dire à la seule critique de l'économie politique. Si cette dimension reste centrale, elle doit selon eux être complétée par le déploiement d'une économie politique du socialisme. C'est pourquoi ils attachent tant d'importance à la lecture d livre III du Capital, et en particulier à la problématique de la transformation des valeurs en prix de production. Ces économistes, pour la plupart membres ou sympathisants de la Ligue communiste révolutionnaire (LCR) ont pour souci de ne pas dissocier analyse théorique et action politique, et inscrivent leurs réflexions et leurs actions militantes dans le cadre de la IV Internationale. Les figures de Jean-Luc Dallemagne et de Jacques Valier doivent être également citées, tant ces économistes ont contribué à la formation et à la diffusion de cette revue.

\section{Diversité des marxismes}

À la périphérie de l'université, parmi les ingénieurs économistes, se déploient deux autres courants de pensée critique s'appuyant sur Marx et les auteurs marxistes. À l'Inra, les économistes lancent un débat autour du statut de l'agriculture dans le capitalisme contemporain, actualisan celui qui fut amorcé par Marx dans Le Capital, prolongé en 1900 par Kautsky (La Question agraire), Rosa Luxemburg et Lénine. Ces auteurs entendaient vérifier si la petite production marchande (en l'occurrence la petite exploitation familiale agricole) allait être absorbée, comme l'avaient prévu Marx et Kautsky, par et dans le mode de production capitaliste, ou bien si l'évolution même du capitalisme allait déboucher sur le maintien de ces formes de production [Cavailhès, 1979].

À la même période s'affirme l'école française de la régulation, dont les figures de proue sont notamment Michel Aglietta, Robert Boyer et Alain Lipietz. Ces économistes se distinguent par leur formation (l'École polytechnique) et par leur appartenance institutionnelle à l'administration économique (INSEE, Direction de la prévision, le CEPREMAP...) au sein de laquelle ils participent de près ou de loin à l'expérience de la planification à la française et à la construction des modèles macro-économétriques. Ils puisent leur vision du capitalisme et leurs outils méthodologiques dans les travaux de l'école « réaliste-sociologique » évoquée plus haut, et produisent une analyse des régimes d'accumulation et des modes de régulation (relations État/économie, régime monétaire, rapport salarial, formes de la 
concurrence formes d'insertion dans la division internationale du travail) qui ponctuent l'histoire du capitalisme. Pour les régulationnistes, une " grande crise capitaliste » traduit l'incapacité du système à maintenir le rythme de l'accumulation du capital et le mode de régulation antérieurs [Aglietta, 1976]. Présents dans les revues académiques en raison du capital symbolique qu'ils détiennent (mathématiques, statistiques, théorie économique...), les régulationnistes bénéficièrent d'une audience universitaire considérable, et d'une visibilité internationale.

Revues, ouvrages, thèses, séminaires, cours et manuels : la référence marxiste a constitué durant les années 1960 et 1970 un point de passage obligé pour les jeunes économistes et pour des cohortes enthousiasmées d'étudiants, insérés par ailleurs dans les dédales du militantisme politique et de la lutte contre l'impérialisme. L'arrivée de la gauche au pouvoir en mai 1981, après près d'un quart de siècle d'opposition, a sans doute sonné comme un aboutissement, la récompense politique de ce déploiement d'énergie intellectuelle.

\section{LE REFLUX DE MARX ET DU MARXISME : IRRÉVERSIBLE OU TEMPORAIRE?}

La visibilité du marxisme en économie au tournant des années 1970 et 1980 apparaît bien trompeuse. Car c'est au moment même où ce courant de pensée paraît triompher que les forces conservatrices se déploient. Tout le cycle idéologique des années 1980 se structure autour de la question des droits de l'homme, de la critique de l'État et de l'utopisme, de la célébration de l'économie libérale et du marché mondialisé. C'est dans ce contexte idéologique que la visibilité du marxisme en économie connaît une érosion certaine. Elle coïncide avec l'affirmation de ce que l'on a rapidemen appelé le «néolibéralisme ». La figure emblématique de cette « révolution conservatrice » est, on le sait, Friedrich von Hayek, lauréat en 1974 du prix de la Banque de Suède pour la science économique (abusivement nommé prix Nobel d'économie). Un combat acharné est livré contre toute forme d'interventionnisme étatique dans les mécanismes de l'économie et, par voie de conséquence, contre le keynésianisme hier triomphant. Marx n'échappe pas à cette lutte pour imposer les idées et les pratiques libérales, en économie comme en politique.

Le déclin par l'intérieur de la science économique

Dans cette conjoncture nouvelle, des économistes qui, hier, travaillaien et diffusaient les idées économiques de Marx, voire les associaient à des perspectives politiques, vont, de l'intérieur, discréditer le marxisme. Deux perspectives politiques, vont, de l'intérieur, discréditer le marxisme. Deux dire, irréversible, au processus de déclin de la pensée critique marxiste. D'abord celui d'Henri Denis, qui, dans L'Économie de Marx. Histoire d'un échec, paru en 1980, poursuit son décorticage des écrits économiques de Marx, et y décèle les contradictions et les limites contenues dans les travaux du philosophe allemand. Elles trouvent leur source selon lui dans les hésitations de Marx vis-à-vis de la dialectique hégélienne, le conduisant à opter in fine pour la théorie de la valeur-travail de Ricardo. Cette lecture, qui revient à classer Marx dans la catégorie des économistes classiques, disqualifie du même coup sa charge critique [Denis, 1980].

L'entreprise consistant à faire de Marx l'héritier direct des économistes classiques, et évacuer la valeur et la plus-value trouve son prolongement dans un second livre, celui de Carlo Benetti et Jean Cartelier, Marchands, salariat et capitalistes, publié en 1981 aux Presses universitaires de Grenoble, dans la collection «Interventions critiques en économie politique » (qui disparaîtra d'ailleurs deux ans plus tard). Benetti et Cartelier voient dans l'économie de Marx une transposition des catégories des classiques que, par ailleurs, il ne cesse de critiquer. Quelques années plus tard, Cartelier, dans un article sur la théorie de la régulation cosigné avec Michel de Vroey, indique qu'il faut renoncer à mettre l'accent sur la critique idéologique de la théorie néoclassique et qu'il convient de se frotter à cette dernière [Benetti, Cartelier, 1981 et Cartelier, de Vroey, 1989]. Du côté de l'école de la régulation, la trajectoire est sensiblement la même. L'évacuation de la théorie de la valeur a constitué l'objectif de Michel Aglietta et d'André Orléan dans La Violence de la monnaie, paru en 1982 Les limites de la valeur les conduisent à construire une théorie de la monnaie fondée non plus sur des rapports sociaux de production, mais sur une approche de la violence telle que ces deux auteurs la trouvent dans l'œuvre de l'anthropologue René Girard [Aglietta et Orléan, 1982 ; Orléan, 2011].

Ces revirements s'inscrivent par surcroît dans un vaste processus de professionnalisation du champ de l'économie, où "le désir de faire science » est de plus en plus structurant pour les économistes [Arena, 2000]. Cet effet de champ se traduit par la formation d'un savoir et d'un langage communs aux économistes du monde entier, leur offrant de plus en plus de prise sur la justification politique de choix économiques, et l'opportunite de ne plus se laisser confiner dans l'enceinte de l'université en pénétrant le marché de l'expertise, ne laissant par voie de conséquence aucune place, ou alors très marginale, au message de Marx. [Lordon, 1997 ; Fourcade, 2009 ; Heredia, 2014]. S'ensuivent alors des procédures de recrutement des enseignants et des critères de publication qui font obstacle à la visibilité de Marx et de sa vision du monde économique. Ce sont ces procédures que contestent aujourd'hui les courants hétérodoxes, qui appellent à davantage de pluralisme dans la science économique. 


\section{LA FIN D'UNE HISTOIRE?}

Ce rapide panorama des relations entre les économistes français et Marx, es années 1950 à aujourd'hui, a permis de montrer que la présence de ' l'auteur du Capital dans le science économique n'avait été qu'un épisode sont délivrés des cours de science ceonomique n'avait éce qu'être refermé relativement court. Le chapitre marxiste en economiesembles'etr. 年 à partir de la fin de la dènes que sous l'influence des acteurs universitaires tiques et sociaur

en-mêmes. économie ? La dynamique même du capitaQu'esperer pour MarX, en économise dans laquelle se trouve la pensée lisme, ses impacts humains et l'impasse dans laquelle se trouve la pens coméconomique dominante incitent les hétérodoxes à persister dans leur combat pour assurer la visibilité de ce penseur hors du commun. Mais on doit bat pour assurer le retour en force de Marx dans les enseignements d'économie

est une hypothèse peu probable, dans un contexte où même les économ

\section{BIBLIOGRAPHIE}

AGt 1976), Régulation et crises du capitalisme. L'expérience des ÉtatsUnis, Calmann-Lévy, Paris.

A LIETTA M. et ORLÉAN A. (1982), La Violence de la monnaie, PUF, Paris.

作 1950 », Revue économique, vol.51, $\mathrm{n}^{\circ} 5$.

La Doctrine économique et sociale de Karl Marx, Seuil, Paris. Vrenoble, Grenoble. BENETTI C. (1976), Valeur et répartition, Presses universitaires de Grenoble, Grenoble. BENETTI C. et CARTELIER J. (1981), Marchands, salariat et capitalistes, Presses universitaires de Grenoble, Grenoble.

RNIS G. De (1966), « Industries industrialisantes et contenu d'une politique d'intégration régionale », Économie appliquée, $\mathrm{n}^{\circ} 3-4$

CETTEL HEIM C. (1964), Planification et croissance accélérée, Maspero, Paris.

BETTELHEIM C. (1964), Planification et croissance acceleré, Me de la rulation : un nouveau paradigme? », Économies et Sociétés, n' 11

paradigme? ", Economies et Societes, CAVAILHÈS J. (1979), Les Réponses des marxistes a la question agromiques de Dijon
de recherche, École nationale superieure des sciences agronomi (Enesad).

COLLECTIF (1971), Traité marxiste d'économie politique. Le capitalisme monopoliste d'État, Éditions sociales, Paris.

DENIS H. (1941), La Corporation, PUF, Paris.
- (1950), La Valeur, Éditions sociales, Paris.

- (1957), « Science marxiste et critique catholique », Économie et Politique, $\mathrm{n}^{\circ} 36$.

- (1966), Histoire de la pensée économique, PUF, Paris.

- (1980), L'Économie de Marx, histoire d'un échec, PUF, Paris.

DENORD F. et ZUNIGO X. (2005), «"Révolutionnairement vôtre". Économie marxiste, militantisme intellectuel et expertise politique chez Charles Bettelheim », Actes de la recherche en sciences sociales, $n^{\circ} 158$.

DUMÉNIL G. et LÉvY D. (2014), La Grande Bifurcation. En finir avec le néolibéralisme, La Découverte, Paris.

FOURCADE M. (2009), Economists and Societies. Discipline and Profession in the United States, Britain and France, 1890s-1990s, Princeton University Press, Princeton.

Heredia M. (2014), À quoi sert un économiste, La Découverte, Paris.

LEBARON F. (2000), La Croyance économique. Les économistes entre science et politique, Seuil, Paris.

LORDON F. (1997), « Le désir de "faire science" », Actes de la recherche en sciences sociales, $\mathrm{n}^{\circ} 119$.

MARCHAL J. (1955), Deux Essais sur le marxisme, Éditions M.-T. Génin, Paris

ORLÉAN A. (2011), L'Empire de la valeur. Refonder l'économie, Seuil, Paris.

POUCH T. (2001), Les Économistes français et le marxisme. Apogée et déclin d'un discours critique (1950-2000), Presses universitaires de Rennes, Rennes.

STEINER P. (2000), « La Revue économique 1950-1980. La marche vers l'orthodoxie académique », Revue économique, $\mathrm{n}^{\circ} 5$. 